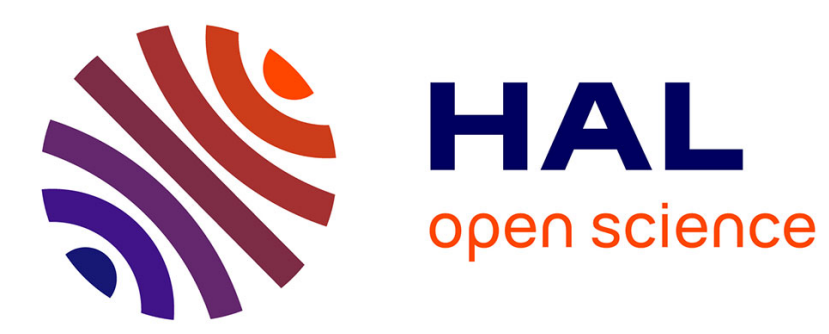

\title{
Adaptive Helmholtz resonator based on electroactive polymers : modeling, characterization, and control
}

Ahmed Abbad, Kanty Rabenorosoa, Morvan Ouisse, Noureddine Atalla

\section{To cite this version:}

Ahmed Abbad, Kanty Rabenorosoa, Morvan Ouisse, Noureddine Atalla. Adaptive Helmholtz resonator based on electroactive polymers: modeling, characterization, and control. Smart Materials and Structures, 2018, 27 (10), 14 p. hal-02130041

\section{HAL Id: hal-02130041 \\ https://hal.science/hal-02130041}

Submitted on 15 May 2019

HAL is a multi-disciplinary open access archive for the deposit and dissemination of scientific research documents, whether they are published or not. The documents may come from teaching and research institutions in France or abroad, or from public or private research centers.
L'archive ouverte pluridisciplinaire HAL, est destinée au dépôt et à la diffusion de documents scientifiques de niveau recherche, publiés ou non, émanant des établissements d'enseignement et de recherche français ou étrangers, des laboratoires publics ou privés. 


\title{
Adaptive Helmholtz resonator based on Electroactive Polymers: Modeling, Characterization, and control
}

\author{
Ahmed Abbad ${ }^{1,2}$, Kanty Rabenorosoa ${ }^{1}$, Morvan Ouisse ${ }^{1}$ and \\ Noureddine Atalla ${ }^{2}$ \\ ${ }^{1}$ Univ. Bourgogne Franche-Comté/CNRS/ENSMM/UTBM - FEMTO-ST Institute, \\ 24 rue de l'Épitaphe, 25000 Besançon, France \\ 2 GAUS, Department of Mechanical Engineering, Université de Sherbrooke, \\ Sherbrooke, QC J1K 2R1, Canada \\ E-mail: ahmed.abbad@usherbrooke.ca
}

\begin{abstract}
This paper presents a new concept and strategy allowing adaptive control of a membraned Helmholtz resonator (HR) embedded in a melamine foam. The designed system aims to adapt the acoustic absorption performances and transmission loss in low frequencies $(<500 \mathrm{~Hz})$. The proposed concept consists in replacing the resonator front wall by an electroactive polymer (EAP) membrane. The stiffness of the membrane can be controlled by an electric field, resulting in a resonance frequency shift. A 2D axisymmetric numerical model based on the finite elements method is developed to characterize the complex structure-acoustic coupling between the membrane, the $\mathrm{HR}$ and the host foam to determine the potential of the concept. Experimental measurements are then performed in an impedance tube and compared to numerical results. A feedforward algorithm based on neural networks allows the adaptivity of the membraned HR to the acoustic excitation variation inside the impedance tube.
\end{abstract}

Keywords: Adaptive Helmholtz resonator, Electroactive polymer (EAP) membrane, Noise control, Acoustic efficiency, Neural network algorithm

\section{Introduction}

Helmholtz resonators (HR) are tunable devices widely used to amplify (e.g. in HI FI speakers) or absorb (e.g. in liners) sounds at a given frequency. They consist of an air filled cavity with an opening (or neck). They can be modeled as a single degree of freedom (SDOF) system with selective resonance frequency related to its neck geometry (associated to the inertial part of the resonator) and the cavity volume which acts as a spring thanks to its compressibility [1]. The mechanisms governing their performances in absorption [2] and transmission loss [3], limited to a single or very narrow frequency band, have been the subject of several studies $[4,5,6]$. Conventional HR have shown weakness when integrated into systems subjected to high frequency variations. Thus, to 
overcome this issue, semi-active systems can provide a way to maintain the efficiency of the resonators to shift the resonance frequency in real time. Adaptive-passive methods have been proposed for narrow frequency band applications: resonators whose tuning frequency is controlled by varying its opening area [7] or its cavity volume [8,9] have been proposed. For the latter, a DC motor controls a movable wall, which modifies its cavity volume. In addition, a feedback based control law is proposed as a tuning strategy. An in-situ, tunable-impedance based on an electromechanical acoustic compliant-backplate HR has been proposed as an alternative [10]. Additional resonances are observed in the impedance curves, leading to interesting sound pressure reduction in the duct. Liu et al. [11] investigated the use of a piezoelectric-composite backplate instead of the isotropic backplates to tune the resonance frequencies. This electromechanical HR was used to control the turbofan engine duct noise. Results show two principal resonance frequencies. The diaphragm geometry (thickness, radius) has been found to have a significant influence on the transmission loss because of the changes in effective cavity volume as a result of the diaphragm motion [12]. An active control is applied to an electronically tunable HR to modify its real impedance using an actuator installed in the resonator is proposed by [13]. It can suppress duct noise at tunable frequencies without increasing the resonator size. The developed control criteria gave a stable and robust active resonator. A theoretically semi-active noise control method (up to 1 $\mathrm{kHz}$ ) for duct-borne transmission noise around various working conditions by the use of a periodic HR in ventilation systems was developed by [14]. The proposed control method combines band-gap attenuation using the side-branch resonators array with the self-adaptive system of noise control. However, the main drawbacks of this type of devices lies in the weight and the complexity of the mechanism which might restrain the practical implementation of these technologies.

In this paper, electroactive polymers (EAPs) [15] have been proposed as solutions to tune a membrane-cavity resonator (EAP membrane coupled with a back cavity). A very common EAP material is made from 3M VHB 4910 (acrylic) [16], however, other silicone-based film have been proposed in the literature [17]. Membranes are typically obtained from stretched dielectric materials where two compliant electrodes (carbon powder, grease,..etc.) are added on both sides of the film. When a high voltage is applied (in the kilo-Volt range), an in-plane expansion results leading to a reduction of the internal stress and change of the tangent stiffness [18, 19, 20]; leading in turn to a change in the membrane natural frequency. Thus, the natural frequencies of a dielectric elastomer can be varied by changing the membrane stretching ratio, or by varying the applied electrical voltage [21]. This electroactive polymer material behavior has already been demonstrated for membranes in different works [22, 23]. The use of EAP has become widespread in the recent years [24, 25]. Many applications in different engineering fields have emerged because of the remarkable features of these materials (lightness, high strain and fast response [26]). Among the most frequent uses, one can mention mechanical actuators, artificial muscles [27], sensors and energy harvester $[28,29]$, but also acoustic actuators [30]. Hence, introducing smart materials in acoustic 
systems offers a real potential for designing adaptive devices. Thus Lu et al. [31] studied the transmission loss performances of a duct silencer using a dielectric membraned cavity. The obtained peaks were tuned by both varying the membrane stretch ratio $(\lambda)$ and the applied voltage. Other works were interested in electroactive membranes based metamaterials. Yu et al. [32] studied a network of four acoustic cavity resonators with a stretching ratio varying from 3.2 to 4 . The peaks observed in the acoustic transmission loss curves can be shifted to lower frequencies when the applied voltage increases. In addition, a broadband control is obtained, showing a promising potential for adaptive noise control in ventilation systems and for other engineering applications. Recently, Yu et al. [33] investigated the acoustic cavity performances installed on a duct and tuned by a dielectric membrane. A three-dimensional analytical model based on the Patch Transfer Function (PTF) method [34] has been developed to characterize complex fluidstructure coupling between the membrane and the surrounding acoustic media. The peaks in the transmission loss curves are shifted to lower frequencies as the applied voltage is increased. Integrating an electroactive membrane on a HR has never been studied before, the main difference with the acoustic cavities consists on the possibility of tuning the "Helmholtz resonance" by varying the applied voltage. In this paper, the upper rigid part of a $\mathrm{HR}$ is replaced by an electroactive membrane and embedded in a melamine foam. Acoustic absorption and transmission loss at normal incidence are experimentally measured and results compared to the finite elements model (FEM). The model takes into account the dissipation mechanism in the foam [35] as well as the membraned HR [36].

Following this introduction, the effect of introducing voltage control to the membrane is investigated through a series of experimental measurements to determine the absorption coefficient as well as the transmission loss of the electroactive membraned HR with two stretch ratios i.e., $\lambda=3$ and 4 (see Section 2). Open-loop measurements are performed to control the resonance frequency shift to determine the limits of the proposed device. The frequency shifting phenomenon is analyzed in both absorption and transmission loss cases. The damping loss factor contribution is studied and a link with the acoustic performances is established. The detailed FE modeling of the proposed resonator is described in Section 3, including the porous treatments, the membrane and acoustic domains. In Section 4, a feedforward control with a neural network algorithm is developed for real-time calculation of the applied voltage giving the desired shift of the resonator.

\section{Adaptive Helmholtz resonator}

The front rigid wall of a HR is replaced by an electroactive membrane as shown in figure 1. The cylindrical cavity is $70 \mathrm{~mm}$ depth and $30 \mathrm{~mm}$ radius. The circular membrane is $30 \mathrm{~mm}$ radius and $0.11 \mathrm{~mm}$ and $0.0625 \mathrm{~mm}$ thick for respectively $\lambda=3$ and $\lambda=4$. The

cylindrical cavity of the $\mathrm{HR}$ is characterized by its depth $\mathrm{l}_{\text {cavity }}$ and radius $\mathrm{r}_{\text {cavity }}$, while the neck dimensions are given by its length $d_{\text {neck }}$ and radius $r_{\text {neck }}$. Values are listed in 
Table 1. Geometrical dimensions of the Helmholtz resonator.

\begin{tabular}{lllll}
\hline & $\mathrm{l}_{\text {neck }}$ & $\mathrm{r}_{\text {neck }}$ & $\mathrm{d}_{\text {cavity }}$ & $\mathrm{r}_{\text {cavity }}$ \\
\hline Dimension $(\mathrm{mm})$ & 30 & 9 & 70 & 30 \\
\hline
\end{tabular}

table 1. The resonator is embedded in a $75 \mathrm{~mm}$ depth and $50 \mathrm{~mm}$ radius ultralight
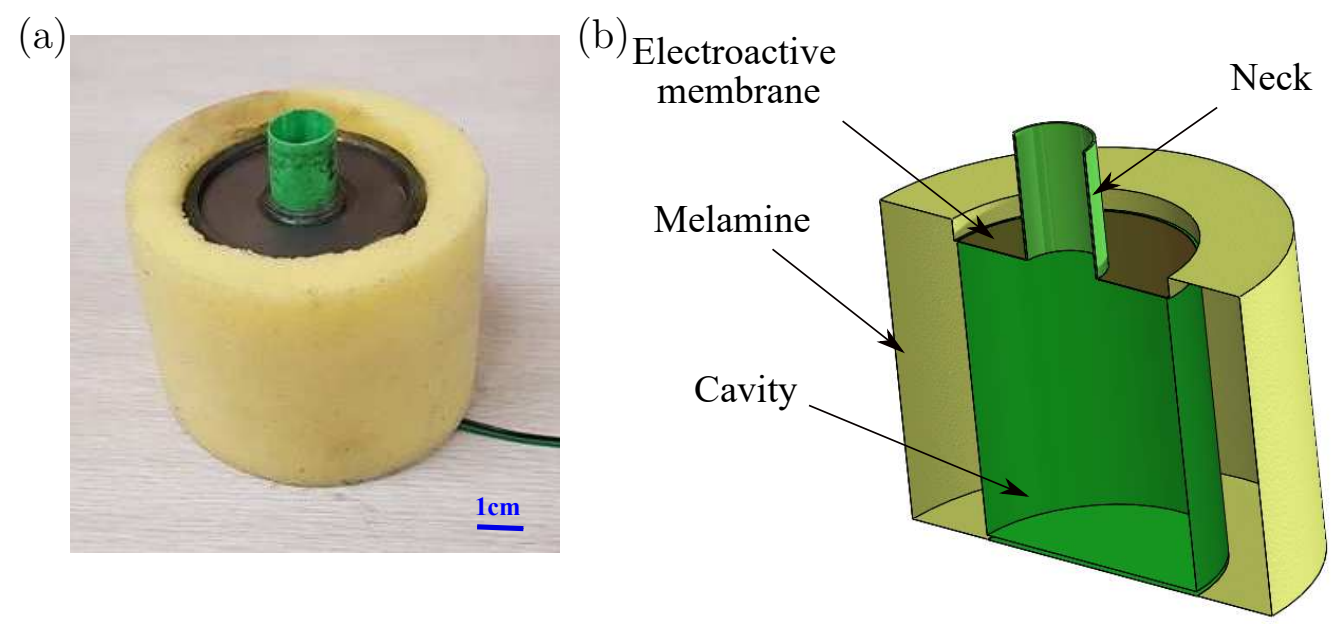

Figure 1. Helmholtz resonator with a front membrane made of electroactive polymer embedded in a melamine foam host support: (a) Real configuration, (b) 3D schematic of the HR components.

melamine foam.

\subsection{Experimental setup}

A loud speaker installed in a $100 \mathrm{~mm}$ diameter tube generates a white noise wave. NI $9263 \mathrm{~A} / \mathrm{D}$ converter $(0-5 \mathrm{~V})$ controls a DC high voltage converter based on EMCO A series generating 0 to $5 \mathrm{kV}$. The three microphones, two cavities method [44] is used to determine the absorption coefficient and transmission loss. Acoustic data acquisitions are operated in the frequency range of interest [100-1200] Hz. The experimental measurement setup is shown in figure 2 .

\subsection{Experimental results}

\subsubsection{Characterization of non active membraned $H R$}

In this section, the membrane is not activated. The contribution of the membraned HR $(\lambda=3$ and $\lambda=4)$ embedded in melamine foam is shown in figures $3(\mathrm{a}, \mathrm{b})$. The figures also show a reference result corresponding to a melamine sample occupying the full volume (i.e., without resonator). The melamine curve obtained from absorption measurement (solid black line) shows a maximum absorption peak of $30 \%$ at $200 \mathrm{~Hz}$ and a resonance at $250 \mathrm{~Hz}$ attributed to the mechanical resonance of the melamine matrix 


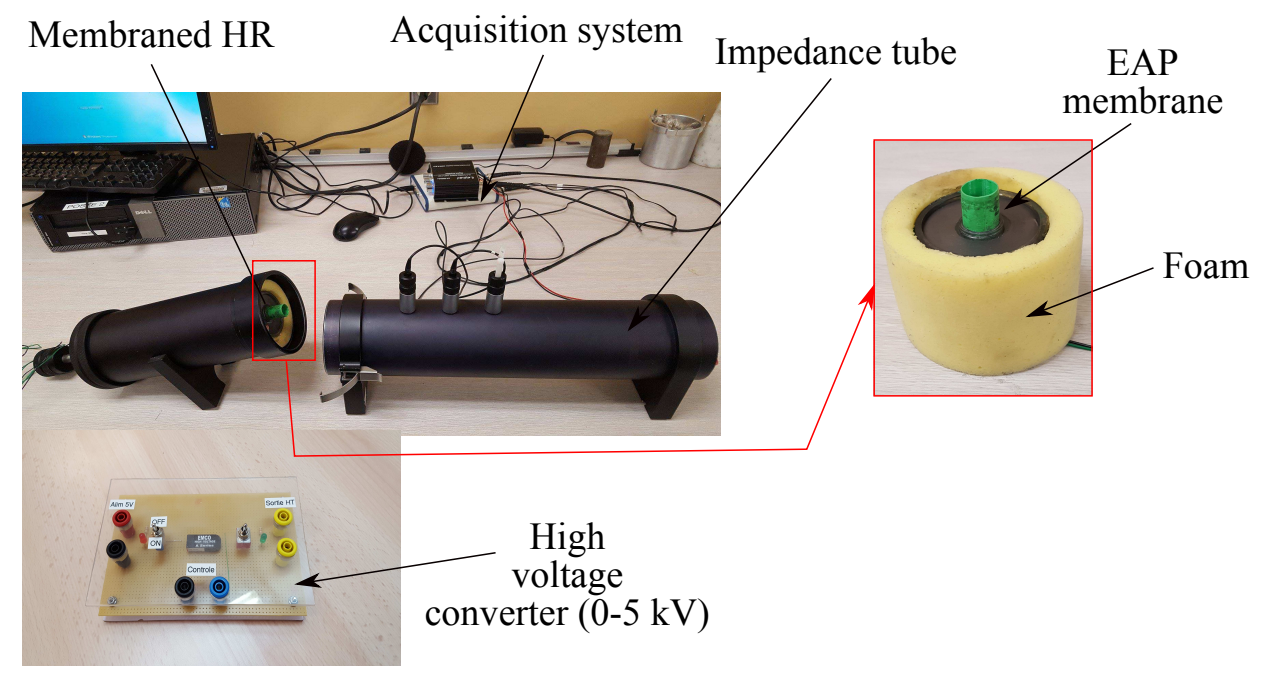

Figure 2. Experimental setup for the characterization of the embedded membraned $\mathrm{HR}$ in a $100 \mathrm{~mm}$ impedance tube.

(Biot frequency). Above this resonance, the foam contributes by $5 \mathrm{~dB}$ for transmission loss in the frequency range 200 to $1200 \mathrm{~Hz}$. The contribution to the sound absorption can be positive or negative at the resonator resonances [35]. The absorption curves of $\lambda=3$ depict one peak at $f=184.5 \mathrm{~Hz}$ and one dip at $f=688 \mathrm{~Hz}$ at the resonance frequencies (respectively $f=186 \mathrm{~Hz}$ and $f=798 \mathrm{~Hz}$ for $\lambda=4$ ) as shown in figure 3 (a), while two peaks are observed for TL (figure $3(\mathrm{~b})$ ). The first peak arises from the Helmholtz resonance modified by the added membrane, which can be considered as an elastic resonator (detailed modeling can be found in [45, 46, 47]). The added elastic wall contributes to decrease the resonator effective compliance and therefore to shift its resonance to lower frequencies. The dip corresponds to the membrane resonance [48]: the membrane is highly damped leading to a large attenuation in transmission and a poor absorption because of the high reactance of the host foam and to the RH filling ratio [35]. The acoustic pressure distribution corresponding the $\lambda=3$ case is computed at $f=184.5 \mathrm{~Hz}$ (which corresponds to the Helmholtz type resonance) and $f=688.5 \mathrm{~Hz}$ (membrane resonance) is shown in figure 3 (c). 
(a)

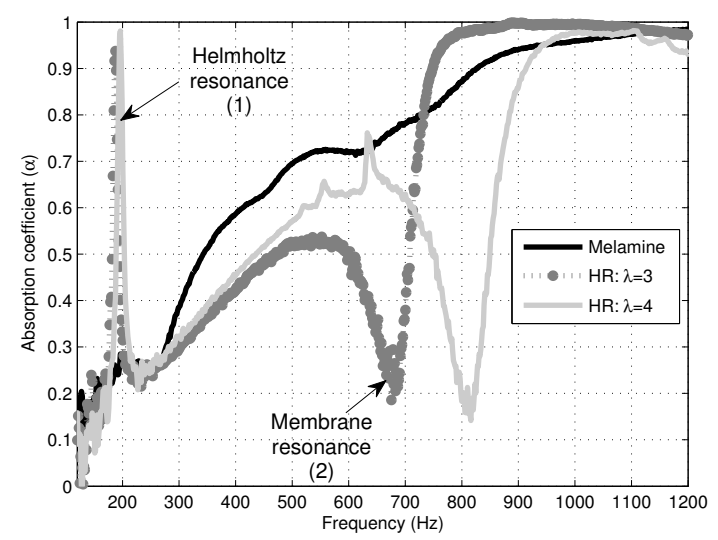

(b)

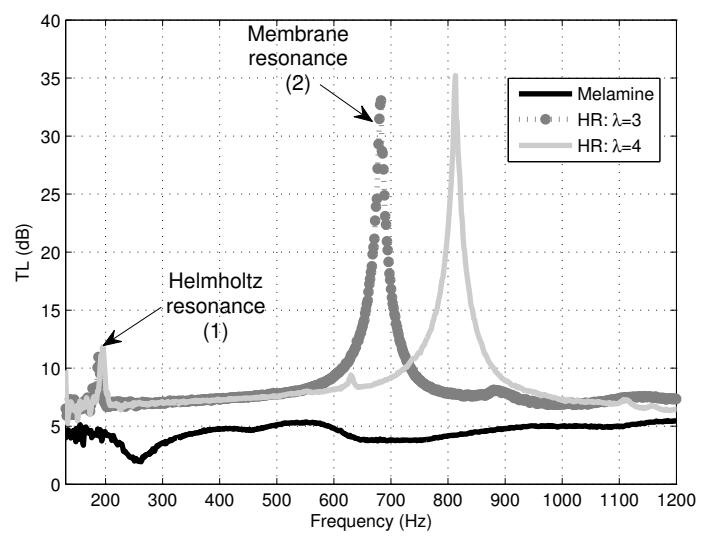

(c)

Figure 3. Experimental (a) absorption coefficient and (b) transmission loss of the membraned HR for $\lambda=3$ and $\lambda=4$ compared to a full melamine foam; (c) Snapshot of the sound pressure field at the two resonances (1) and (2).

At resonances, the sound pressure observed inside the resonator is much larger than in the rest of the tube, demonstrating, thus, the ability of the device to attenuate certain frequencies by localizing the acoustic energy in the resonator.

The resonator enhances the sound transmission loss of the melamine foam because of the added mass and the two resonances. A first peak of $9 \mathrm{~dB}$ amplitude is observed at the first resonance frequency, beside, the second peak improves the transmission loss efficiency with a $33 \mathrm{~dB}$ amplitude.

The next section discusses the open-loop results for different applied voltages.

\subsubsection{Static identification}

The application of the electrical voltage $V$ [Volt] changes the EAP membrane stiffness and therefore its mechanical and acoustical properties. Variations of the sound absorption and transmission loss are obtained. Analysis of this effect is carried out in an open-loop in the section. The applied electric voltage varies from 0 to $5 \mathrm{kV}$ and the sound absorption $(\alpha)$ and TL are investigated. Results are shown in figure 4 (a, 
b). The acoustic device depicts one peak and one dip at the resonance frequencies

(a)

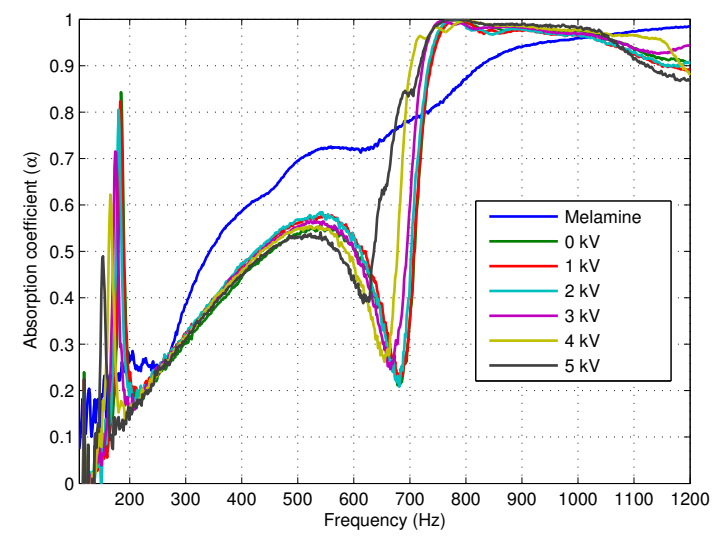

(b)

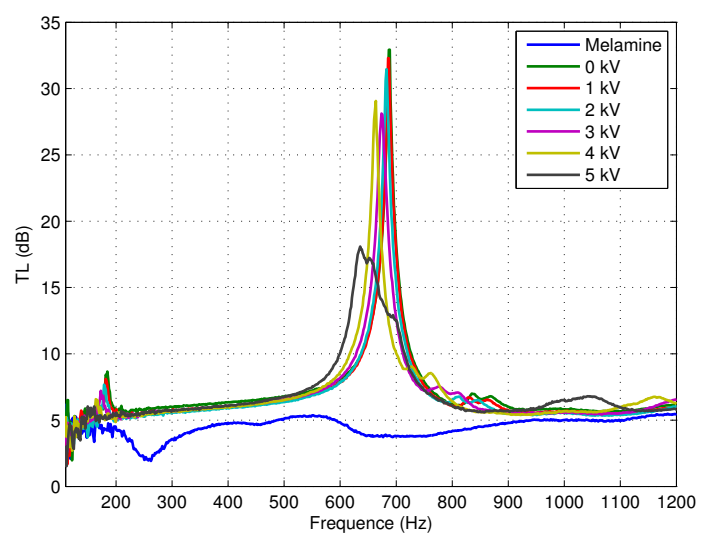

Figure 4. Experimental (a) absorption coefficient and (b) transmission loss for various voltage values for $\lambda=3$ both compared to a full melamine foam.

on the absorption curve, while two peaks are observed in the case of transmission loss. As expected, the variation of the electrical voltages induces a to shift of the

Table 2. First and second resonances as function of the applied voltage for $\lambda=3$.

\begin{tabular}{ll|llllll}
\hline & & & & & & \\
$f_{r}(\mathrm{~Hz})$ & 0 & 1 & 2 & 3 & 4 & 5 \\
\hline$f_{\text {res } 1}$ & 184.5 & 183.0 & 180.0 & 174.5 & 165.5 & 152.5 \\
$f_{\text {res } 2}$ & 688.5 & 687.0 & 682.6 & 673.8 & 663.6 & 635.7 \\
\hline
\end{tabular}

resonances of the resonators. The peak shifts are explained by the interactions between the electroactive membrane of variable stiffness (equation (5)) and the resonances of the cavity. Absorption coefficient curves for the electroactive membrane Helmholtz resonator $(\lambda=3)$ are plotted in figure 4 (a). Outside the resonance frequencies, the Helmholtz resonator behavior is independent from the electrical voltage. The first zero voltage resonance is observed at a frequency of $184.6 \mathrm{~Hz}$ with an amplitude of 0.85 . For the maximum voltage of $5 \mathrm{kV}$, this frequency is lowered to $152.5 \mathrm{~Hz}$ with an amplitude of 0.5 . Hence, $32 \mathrm{~Hz}$ offset is obtained (around $20 \%$ ). On the transmission loss curves (figure 4 (b)), two peaks are observed. The application of a voltage has the same effect as for the absorption coefficient, since a frequency shift is observed. For a null voltage, the first peak has an amplitude of $8.7 \mathrm{~dB}$. It is reduced to $6 \mathrm{~dB}$ for $5 \mathrm{kV}$. The second resonance has an amplitude around $33 \mathrm{~dB}$. This value decreases to $17.9 \mathrm{~dB}$ when $5 \mathrm{kV}$ is applied. All resonances frequencies values are listed in table 2.

The shift of the first resonance as function of the applied voltage is listed in the following table 3 . 
Table 3. First resonance frequency $\left(f_{\text {res } 1}\right)$ shift as function of the applied voltage for $\lambda=3$.

\begin{tabular}{lllllll}
\hline Voltage $(\mathrm{kV})$ & 0 & 1 & 2 & 3 & 4 & 5 \\
\hline Frequency shift $(\mathrm{Hz})$ & 0 & 1.5 & 4.5 & 10 & 19 & 32 \\
\hline
\end{tabular}

(a)

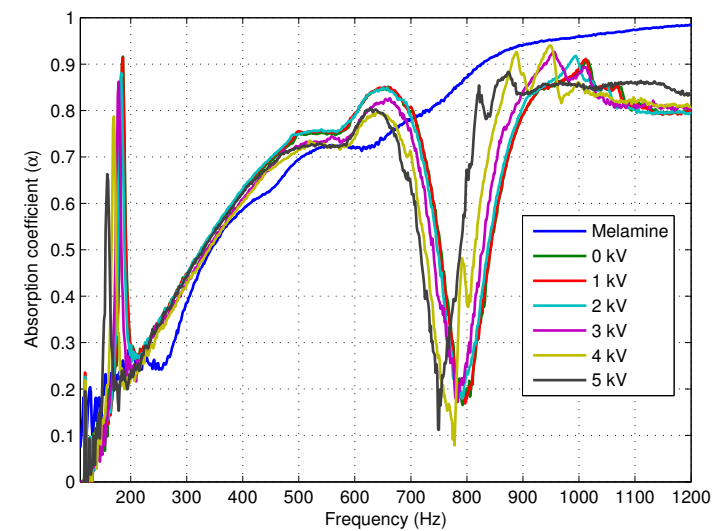

(b)

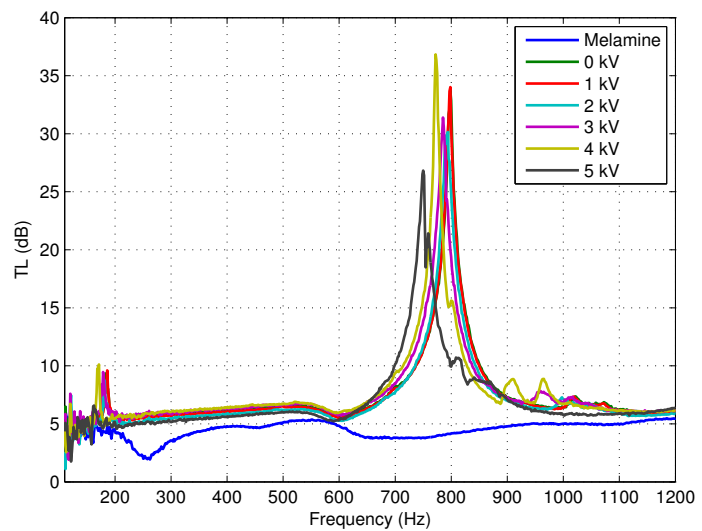

Figure 5. Experimental (a) absorption coefficient and (b) transmission loss as function of the applied voltage variation for $\lambda=4$ both compared to the full melamine foam.

Table 4. First and second resonances as function of the applied voltage for $\lambda=4$.

\begin{tabular}{|c|c|c|c|c|c|c|}
\hline$f_{r}(\mathrm{~Hz})$ & 0 & 1 & 2 & 3 & 4 & 5 \\
\hline$f_{\text {res } 1}$ & 186 & 186 & 183 & 179 & 170 & 158 \\
\hline$f_{\text {res } 2}$ & 798 & 798 & 792 & 785 & 772 & 750 \\
\hline
\end{tabular}

The expected effect is thus clearly observed: a shift towards the low frequencies of the first resonance results from the increase in the electrical voltage. It should be noted that this property of interest results in a reduction in efficiency, whether in terms of absorption or transmission. A similar analysis for $\lambda=4$ leads to identical conclusions. The values of the resonant frequencies are listed in table 4 . The first resonance frequency $(0 \mathrm{kV})$ is $186 \mathrm{~Hz}$, higher than that for $\lambda=3(184.6 \mathrm{~Hz})$ with a tiny difference of just 1.4 Hz. The gap becomes larger for the second resonance with $798 \mathrm{~Hz}$ for $\lambda=4$ and $688.5 \mathrm{~Hz}$ for $\lambda=3$. This difference of $109.5 \mathrm{~Hz}$ is justified by the fact that this resonance is due to the membrane vibration which has a smaller thickness for $\lambda=4(0.0625 \mathrm{~mm})$ than in the $\lambda=3(0.11 \mathrm{~mm})$. For $\lambda=4$, the first resonance of the absorption curve has an amplitude of $90 \%$. For $5 \mathrm{kV}$, a shift of $32 \mathrm{~Hz}$ and an amplitude drop of $66 \%$ are observed. For transmission loss (figure $5(\mathrm{~b})$ ), peaks amplitudes are generally higher than ones obtained for $\lambda=3$. The amplitude falls to near extinction at $5 \mathrm{kV}$ is due to the loss factor increase 
Table 5. Frequency shift as function of the applied voltage for $\lambda=4$.

\begin{tabular}{lllllll}
\hline$V(\mathrm{kV})$ & 0 & 1 & 2 & 3 & 4 & 5 \\
\hline Frequency shift $(\mathrm{Hz})$ & 0 & 0 & 3 & 7 & 16 & 32 \\
\hline
\end{tabular}

resulting from the applied voltage. The different mechanisms related to resonances, and the loss factor increase are studied in section 2.3. With regard to the second resonance, the transmission loss efficiency remains high, the resonant frequency is shifted, which is synonymous with a change in the electroactive membrane internal stress, without necessarily bringing a large change in peak amplitudes except in the case of $5 \mathrm{kV}$. Therefore, the stretch ratio $\lambda$ affects the absorption coefficient and the transmission loss of the resonator. The first frequencies (Helmholtz resonances) are quite close comparing $\lambda=3$ and 4 results, the difference is more apparent for the second (membrane) resonances. For possible applications, the targeted resonant frequencies determine the choice of the stretch ratio to use. Having found the possibility of controlling the resonator behavior by varying the applied voltage, the next section is focused on the validation of the numerical model.

\subsection{Membrane damping analysis}

Phenomenon linked to the absorption (respectively the transmission loss) amplitude fall noticed during the resonance shift towards the low frequencies are investigated. Experimental setup is shown in figure 6. The membraned HR are settled on a frame itself fixed on an impedance tube, a white noise excitation is generated by a speaker installed in the end of the tube in which a reference microphone is located. A 1D laser vibrometer (Doppler Polytec-PDV100) measures the membrane velocity, the transfer function between the membrane velocity and the reference pressure is thus established. The analysis is carried out to determine the effect of the membrane damping on the resonator performances. The fall in amplitude has already been mentioned in the literature: Dubois [19] studied the electrical voltage effect on the membrane (made from Sylgard 184 PDMS) resonance frequency with a resonance drop of up to $40 \%$; Hochradel [30] reported the resonances dependency of dielectric elastomer actuators such as VHB 4905 on the applied voltage, $30 \%$ shift is observed for $3 \mathrm{kV}$; but to our knowledge, no identification of damping of EAP membranes under applied voltage is available in literature. 


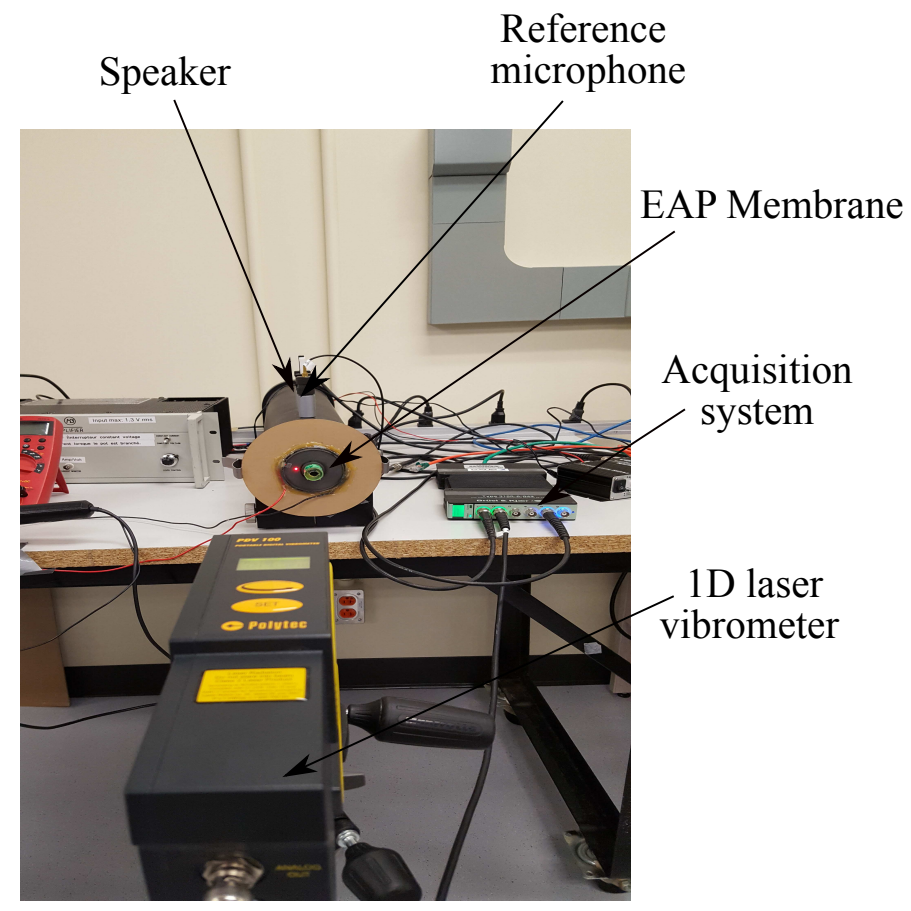

Figure 6. Experimental setup for measuring the velocity of the electroactive membranes.

The loss factors $\eta$ are determined by the $-3 \mathrm{~dB}$ bandwidth method applied on the measured transfer function. figure 7 illustrates the identification of the first mode of the

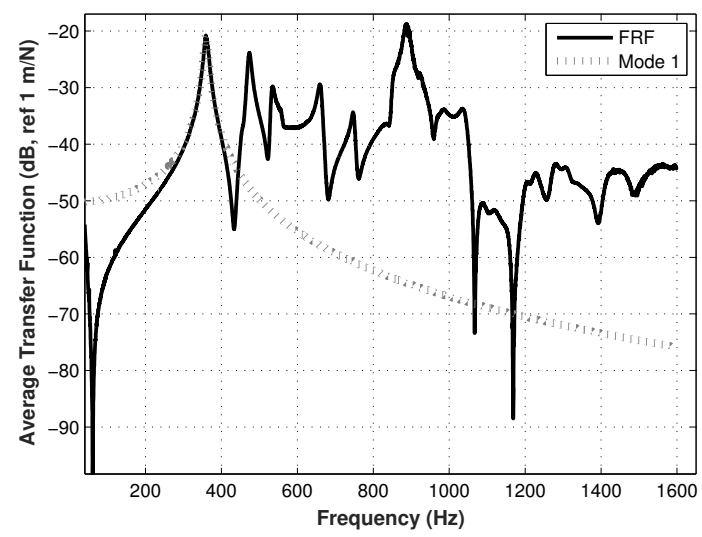

Figure 7. Identification of the first eigenmode's loss factor $\eta$ of the electroactive membrane for a null voltage $(\lambda=3)$.

zero voltage membrane $(\lambda=3)$. This latter step is repeated for voltage values from 1 to $5 \mathrm{kV}$.

The obtained loss factor $\eta$ values are listed in table $6, \eta$ increases with the applied voltage. This trend is consistent with previous observations on the absorption factor and TL measurements: whether it is the first or the second resonance, an amplitude 
drop is observed for the two resonators $(\lambda=3$ and $\lambda=4)$ when the voltage increases. The

Table 6. Variation of the loss factor $\eta$ of the electroactive membrane as a function of the applied voltage for $\lambda=3$ and $\lambda=4$.

\begin{tabular}{ll|llllll}
\hline \multicolumn{1}{l}{$V(\mathrm{kV})$} & 0 & 1 & 2 & 3 & 4 & 5 \\
\hline$\eta_{\lambda=3}$ & 0.020 & 0.030 & 0.036 & 0.038 & 0.040 & 0.050 \\
$\eta_{\lambda=4}$ & 0.030 & 0.031 & 0.031 & 0.034 & 0.034 & 0.045 \\
\hline
\end{tabular}

loss factor $\eta$ contributes to lower the peak amplitudes of the absorption coefficients. The study allows to establish a link between the frequency shift and the amplitude fall. Observations made by Zhu [21] concerning the reduction of the resonant frequencies with the applied voltage are confirmed, this is explained by the increase of the membrane stiffness.

\section{Numerical model}

A 2D axisymmetric numerical model capable of describing the structuralacoustic coupling is developed in order to predict the acoustic performances of the proposed device. A schematic of the geometry in the calculation is shown in figure 8 . The numerical model simulates a $100 \mathrm{~mm}$ diameter impedance tube test for the measurement of the absorption coefficient and the transmission loss on frequency range 100 to 1200 $\mathrm{Hz}$. At the impedance tube walls, a rigid wall boundary condition is imposed in the longitudinal direction. The mesh is based on quadratic triangular elements for the tube, the foam and the resonator. The element size is chosen in order to have 6 elements per wavelength on the whole model [37]. A multiphysics modeling of the foam as well as HR are needed. The resonator cavity walls are considered rigid as well as for the neck, the melamine foam behavior and the viscous loss in the resonator neck are modeled using the Johnson-Champoux-Allard model (JCA) model [35]. In addition the stretched membrane is modeled in the model by considering its in-plane force for different electrical voltage values. 


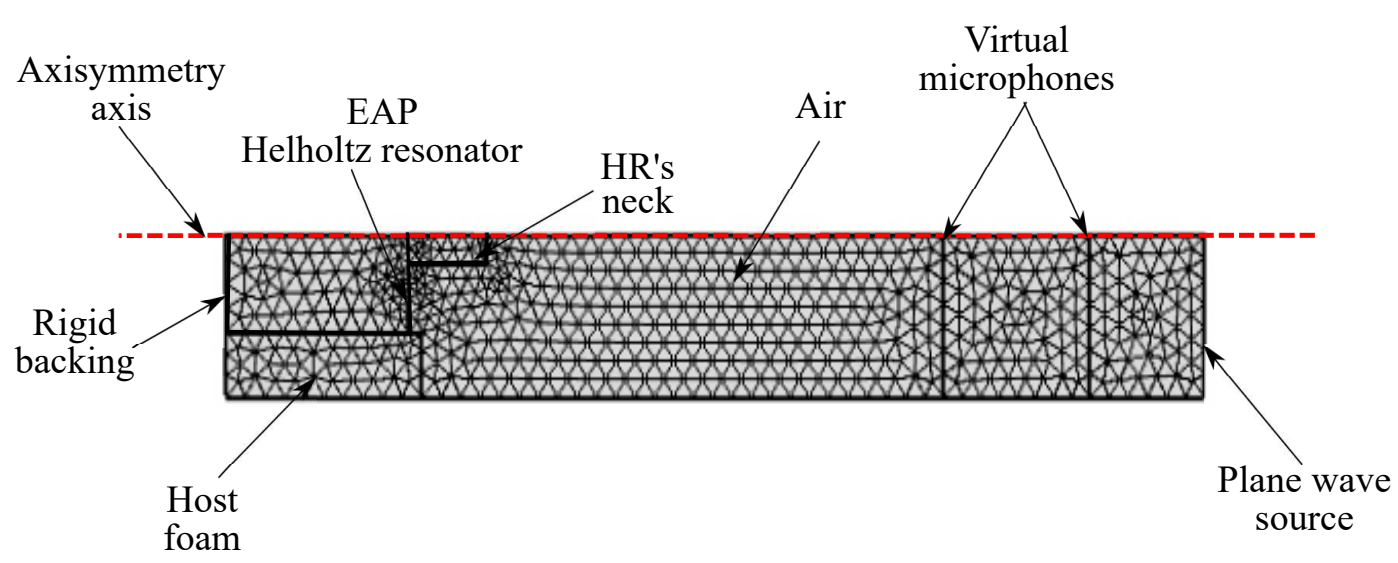

Figure 8. Geometry of meshes used in the finite element model with plane wave source at one end and rigid backing at the opposite end. The two microphones are used to estimate absorption coefficient of the membraned HR embedded in melamine foam.

\subsection{Acoustic model}

The acoustic fields inside the tube and resonators cavities are described by Helmholtz's equation

$$
\Delta p+k^{2} p=0, \quad k^{2}=\frac{\omega^{2}}{c_{0}^{2}},
$$

where $p$ is the acoustic pressure, $k$ is the wave number and $c_{0}=340 \mathrm{~m} / \mathrm{s}$ the sound speed in air.

The melamine foam is modeled as an equivalent fluid with a rigid frame based on the Johnson-Champoux-Allard model (JCA) [38]. This model is described by the Helmholtz equation in which the squared sound speed $c_{0}^{2}$ is replaced by $K_{\text {eff }} / \rho_{\text {eff }}$, where $K_{\text {eff }}$ and $\rho_{\text {eff }}$ are respectively the frequency-dependent effective bulk modulus and effective density. The JCA model is based on five intrinsic experimentally measured properties i.e., the flow resistivity $\sigma$, the porosity $\Phi$, the tortuosity $\alpha_{\infty}$, the viscous $\Lambda$ and thermal $\Lambda^{\prime}$ characteristic length. The intrinsic properties given in table 7 are obtained experimentally from the measurements carried out on the porous materials characterization benches at GAUS (Universit de Sherbrooke). The visco-thermal losses in the resonator neck are considered by using its effective complex and frequency dependent parameters [39] based also on the JCA model: the neck is assimilated to a cylindrical pore [40]. where corresponding parameters are given as $\Phi_{\text {neck }}=1, \alpha_{\text {neck }}=1$, $\sigma_{\text {neck }}=\frac{8 \eta_{\text {air }}}{r_{\text {neck }}^{2}}\left(\eta_{\text {air }}\right.$ being the air viscosity $)$, and $\Lambda_{\text {neck }}=\Lambda_{\text {neck }}^{\prime}=r_{\text {neck }}$.

3.1.1. Membrane modeling A structural FE model was developed in order to determine the membrane in-plane force $T_{0}$. This latter is a key parameter to adapt the acoustic device performances. Ogden model with experimentally determined parameters (listed in table 8) is used to describe the hyperelastic material model [41]. In the present $N$ parameters model, the first Piolla-Kirchoff stress tensor $P_{1}$ of the material is given in 
Table 7. Johnson-Champoux-Allard model parameters of the ultralight melamine foam identified experimentally.

\begin{tabular}{lllll}
\hline $\begin{array}{l}\sigma \\
\left(\mathrm{N} . s . \mathrm{m}^{-4}\right)\end{array}$ & $\Phi$ & $\alpha_{\infty}$ & $\begin{array}{l}\Lambda \\
(\mu \mathrm{m})\end{array}$ & $\begin{array}{l}\Lambda^{\prime} \\
(\mu \mathrm{m})\end{array}$ \\
\hline 8432 & 0.99 & 1.02 & 138 & 154 \\
\hline
\end{tabular}

Table 8. Parameters of the 3M VHB 4910 acrylic described by Ogden hyperelastic model.

\begin{tabular}{ll}
\hline Parameter & Value \\
\hline$\mu_{1}[\mathrm{kPa}]$ & 104.5 \\
$\alpha_{1}[-]$ & 0.93 \\
$\mu_{2}[\mathrm{kPa}]$ & 0.0529 \\
$\alpha_{2}[-]$ & 5.38 \\
\hline
\end{tabular}

terms of the stretch ratio $\lambda$ and the empirically determined Ogden parameters $\mu_{i}$ and $\alpha_{i}$.

$$
P_{1}=\sum_{i=1}^{N} \mu_{i}\left(\lambda^{\alpha_{i}-1}-\lambda^{-\frac{\alpha_{i}}{2}-1}\right) .
$$

The $t_{0}=1 \mathrm{~mm}$ thick square $\left(l_{x}^{0}=l_{y}^{0}=10 \mathrm{~cm}\right)$ membrane is stretched 3 and 4 times in both $X$ and $Y$ directions. The obtained thickness in the stretched state $t_{s}$ is calculated by

$$
t_{s}=t_{0} / \lambda^{2},
$$

where $\lambda=l_{x}^{s} / l_{x}^{0}, l_{x}^{s}$ being the membrane length after stretching. The obtained thicknesses are $0.11 \mathrm{~mm}$ and $0.0625 \mathrm{~mm}$ for $\lambda=3$ and $\lambda=4$, respectively. Simulation yields an in place force of $T_{0}=30.5 \mathrm{~N} / \mathrm{m}$ for $\lambda=3$ and $26.4 \mathrm{~N} / \mathrm{m}$ for $\lambda=4$.

Introducing a voltage control to the membrane, in pursuing a possible tuning of the resonator acoustic performances is investigated. When a voltage is applied between the two electrodes, a Maxwell pressure is created upon the dielectric layer. The membrane become thinner, expends in the planar directions and increases its surface, causing a decrease in its internal stress $\sigma$ and therefore the in-plane force $T_{0}$. Thus, the voltage effect is taken into account according to [19]

$$
\sigma_{p}=\sigma_{0}-\varepsilon \frac{V^{2}}{t_{s}^{2}},
$$

where $\sigma_{p}$ is the membrane stress due to the applied voltage, $\sigma_{0}$ is the membrane initial stress, $\varepsilon$ the elastomer relative permittivity [42] and $t_{s}$ the membrane thickness. 
Equation (4) is equivalent to

$$
T_{p}=T_{0}-\varepsilon_{p} V^{2}
$$

where $T_{0}$ and $T_{p}$ are respectively, the membrane in-plane force before and after applying

the voltage, $\varepsilon_{p}=\frac{\varepsilon}{t_{s}}$ values are determined based on the linear regression function.

$T_{p}$ values for 0,3 and $5 \mathrm{kV}$ are first obtained numerically by identification process based on the Least Mean Square method, then a linear regression function is used in order to generalize the evolution of $T_{p}$ as a function of the applied voltage $V$ [33].

The acoustic fields inside the tube, the resonator cavity and neck satisfy the Helmholtz's equation, the circular membrane is governed by its dynamic equation of flexural vibration, which amplitude $w(x, y)$ under harmonic difference pressure load $\Delta p$ at angular frequency $\omega$ is governed by equation 6

$$
T_{0} \nabla^{2} w(x, y)+\omega^{2} t_{s} \rho_{m} w(x, y)=\Delta p
$$

The density of the 3M VHB 4910 is $960 \mathrm{~kg} / \mathrm{m}^{3}$ following [16], while Poisson's ratio is 0.49. The damping effect is taken into account in the FE model by using a complex inplane force $T_{0}^{*}=T_{0}(1+j \eta)$ with $\eta$ the membrane loss factor determined experimentally as detailed in Section 2.3, values are listed in table 6 .

\subsection{Acoustic indicators}

The aim of the model is to estimate two acoustic indicators: absorption coefficient $\alpha$ and transmission loss (TL). Two virtual microphones are used to determine the magnitude of the forward and backward traveling waves, from which sound absorption coefficient may be determined. It is assumed that only plane waves propagates in the tube from the right end and the specimen being in the other end. The sound absorption coefficient $\alpha$ is defined as the ratio of sound power absorbed by a surface to the incident sound power [43]. The sound absorption coefficient for a plane wave at normal incidence is obtained from the reflection coefficient $R$, and is given by

$$
\alpha=1-|R|^{2}
$$

The acoustic attenuation capability, which is often refereed to as TL in decibels is one of the most important factors in designing acoustic resonators. Hence, an anechoic termination condition $Z=\rho_{0} c_{0}\left(\rho_{0}=1.213 \mathrm{~kg} / \mathrm{m}^{3}\right.$ is the air density and $c_{0}=340 \mathrm{~m} / \mathrm{s}$ is the sound velocity in air) is applied at the end of the tube and the average pressures on two parallel surfaces to the acoustic excitation in upstream $p_{1}$ and downstream $p_{2}$ of the tested devices are raised. Transmission loss is determined as

$$
\mathrm{TL}=20 \log _{10}\left(\frac{p_{2}}{p_{1}}\right) .
$$




\subsection{EAP membrane behavior}

The EAP membrane is described by equation (5). The model being quite simple and sensitive to mounting conditions, a linear regression is performed to identify two models (for $\lambda=3$ and $\lambda=4$ ) allowing correct estimation of the resonance frequencies identified in tables 2 and 4 . These models are:

$$
T_{p}^{\lambda=3}=T_{0}^{\lambda=3}-0.48 V^{2}
$$

and

$$
T_{p}^{\lambda=4}=T_{0}^{\lambda=4}-0.4 V^{2} .
$$

The value of the membrane in-plane force $T_{p}$ as function of the applied voltage obtained both from numerical and linear regression approach are reported in figure 9 . The evolution is non-linear with a tendency which is in adequacy with the two equations (9) and (10). $T_{p}$ and $V$ units used in the above equations are kilo-pascal (kPa) and kilo-volt $(\mathrm{kV})$, respectively. For zero voltage, the membrane in-plane force values are $T_{0}=29 \mathrm{~N} / \mathrm{m}$ for $\lambda=3$ and $T_{0}=28 \mathrm{~N} / \mathrm{m}$ for $\lambda=4$. These values are obtained from identification process to fit the experimental measurements. They are lower compared to those obtained from the stretch process FEM simulation $\left(T_{0}=30.5 \mathrm{~N} / \mathrm{m}\right.$ for $\lambda=3$ and $T_{0}=26.4 \mathrm{~N} / \mathrm{m}$ for $\left.\lambda=4\right)$. This adjustment can be explained considering the added electrodes (which is not taken into account in the FE model) and the membrane-frame assembly process subject to experimental uncertainties.

The figure 9 shows the identified values of the membrane in-plane forces and the corresponding regressions. The values of the membrane in-plane force $T_{p}$ as function of

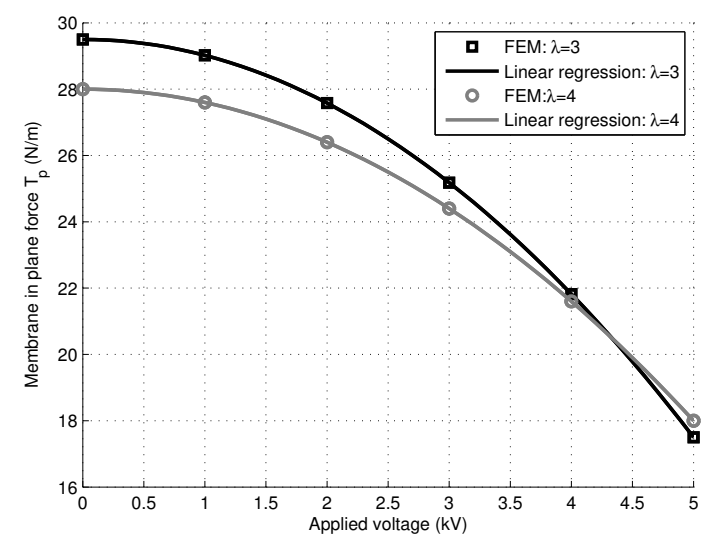

Figure 9. Membrane in-plane force $T_{p}$ as function of the applied electric voltage $(V)$ for $\lambda=3$ and $\lambda=4$ : linear regression result compared to the numerical model.

the applied voltage $V$ for $\lambda=3$ and $\lambda=4$ are listed in table 9 . In the following, these models are used to estimate the effect of the applied voltage on the absorption coefficient factor and TL curves. Results are presented for $2 \mathrm{kV}$ and $5 \mathrm{kV}$ only. 
Table 9. Membrane in-plane force $T_{p}$ as function of the applied electric voltage $V$ for $\lambda=3$ and $\lambda=4$.

\begin{tabular}{lc|cccccc}
\hline & $V(\mathrm{kV})$ & 0 & 1 & 2 & 3 & 4 & 5 \\
\hline$T_{p}^{\lambda}(\mathrm{N} / \mathrm{m})$ & & 29.0 & 29.0 & 27.6 & 25.2 & 21.8 & 17.5 \\
\hline$T_{p}^{\lambda=3}$ & 28.0 & 27.6 & 26.4 & 24.4 & 21.6 & 18.0 \\
$T_{p}^{\lambda=4}$ & & &
\end{tabular}

\subsection{Results}

\subsubsection{Absorption}

In figure 10 (a, b), a good agreement between numerical and experimental curves for $\lambda=3$ is observed, the vibroacoustic behavior is faithfully described and resonance peaks are well estimated. For $\lambda=4$ (figure 11 (c, d)), the numerical model predicts the two

(a)

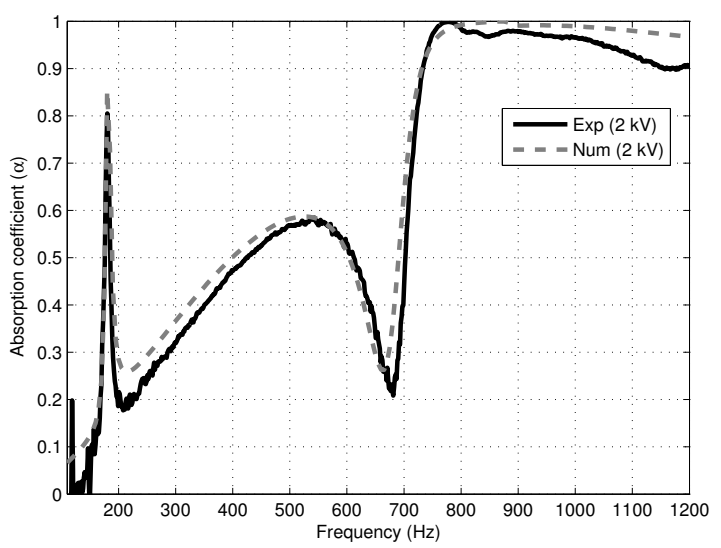

(b)

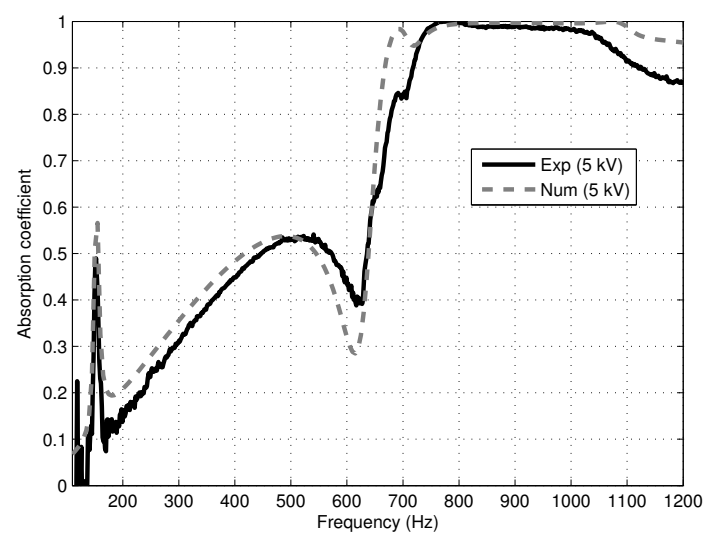

Figure 10. Absorption coefficients of the electroactive Helmholtz resonator: comparison of results from experimental and numerical model. (a) $\lambda=3$ for $2 \mathrm{kV}$, (b) $\lambda=3$ for $5 \mathrm{kV}$.

main resonances of the system, while a mean frequency difference is observed over the interval [200-700] Hz, which could be attributed to the uncertainties during experiments (resonator dimensions and it's mounting on the foam). 
(a)

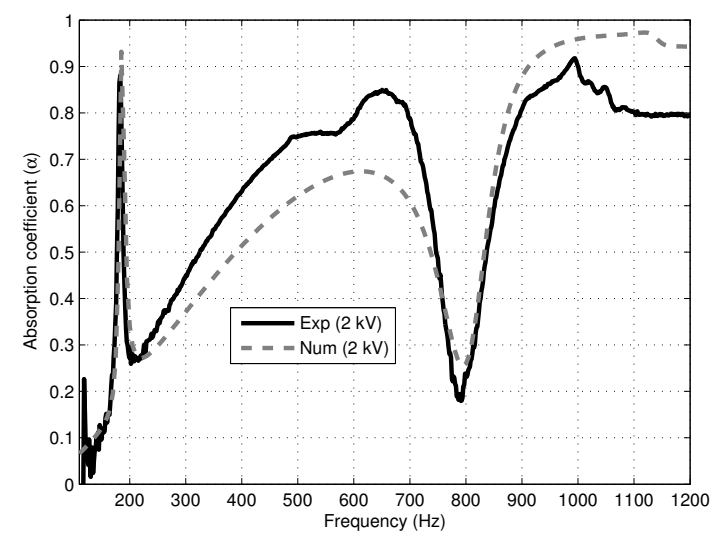

(b)

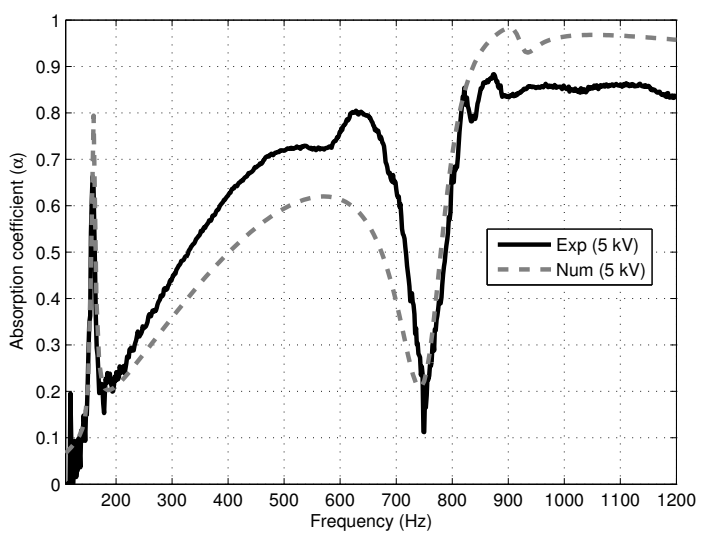

Figure 11. Absorption coefficients of the electroactive Helmholtz resonator: comparison of results from experimental and numerical model. (a) $\lambda=4$ for $2 \mathrm{kV}$ and (b) $\lambda=4$ for $5 \mathrm{kV}$.

\subsubsection{Transmission loss}

Transmission loss results for $\lambda=3(2 \mathrm{kV}$ and $5 \mathrm{kV})$ are compared in figure $12(\mathrm{a}, \mathrm{b})$. It is observed that numerical model fits well with the experimental data, peak resonances and amplitudes are correctly predicted with small differences for $2 \mathrm{kV}(0.8 \%$ for the second resonance). This observation is similar to that made on the absorption curves. The differences are mainly due to experimental uncertainties. 
(a)

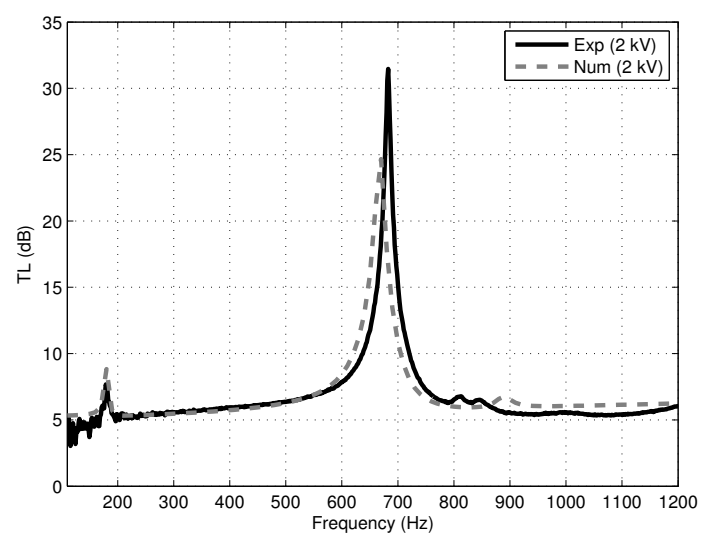

(c)

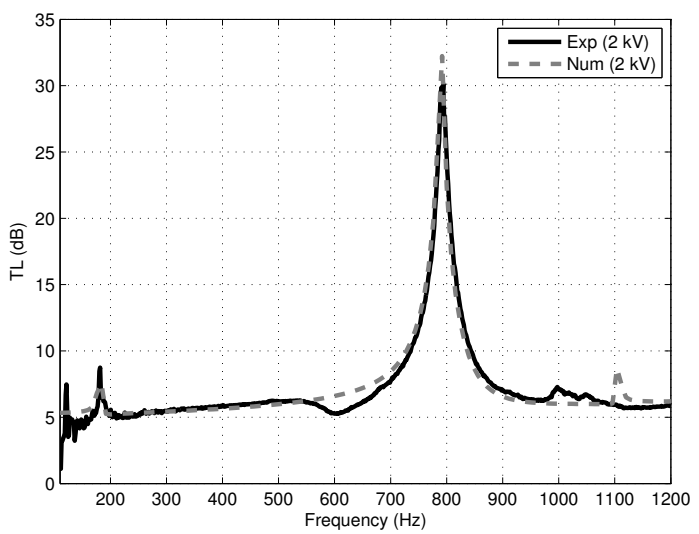

(b)

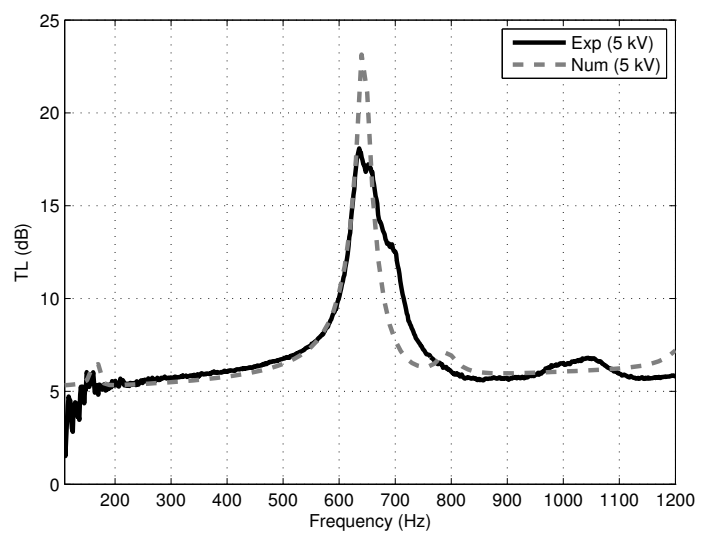

(d)

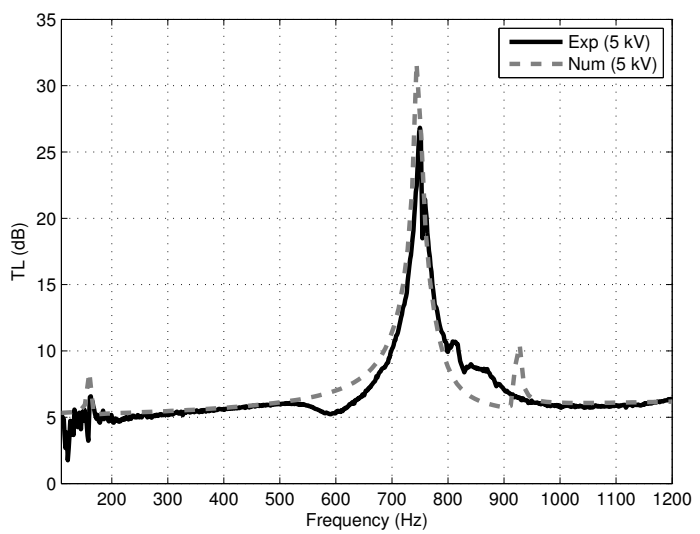

Figure 12. Transmission loss of the electroactive Helmholtz resonator: comparison of results from experimental and numerical model. (a) $\lambda=3$ for $2 \mathrm{kV}$, (b) $\lambda=3$ for $5 \mathrm{kV}$, (c) $\lambda=4$ for $2 \mathrm{kV}$ and (d) $\lambda=4$ for $5 \mathrm{kV}$.

For $\lambda=4$ (figure $12(\mathrm{c}, \mathrm{d})$ ); the numerical model allows to estimate the two main resonances of the resonator for $2 \mathrm{kV}$ and $5 \mathrm{kV}$ voltages. It accurately describes the transmission loss behavior of the device. As concluded previously, the variation of the electrical voltage allows to shift the resonances towards the low frequencies. The obtained results show the potential offered by such devices for the control of resonances of the HR through an electromechanical system based on electroactive membrane.

In the next section, a feedforward control algorithm is proposed to study the device's ability to adapt to a tonal noise excitation variation.

\section{Closed loop control based on neural network algorithm}

Shifts observed in open-loop measurements have allowed to assess the electroactive membrane resonator ability to control the resonance frequency. Therefore, a Feedforward based algorithm is proposed and experimentally tested. The control law is based on the Neural Networks (NN) algorithm of Matlab toolbox [49]. In the present case, the NN is composed of 10 neurons and 2 layers. The training of the NN is based 
on the optimization method of Levenberg-Marquardt combined with the Least Mean Square (LMS) performance function to better approximate the nonlinear function fit problems [50].

\subsection{Control command}

The control law is built for the two electroactive HRs $(\lambda=3$ and $\lambda=4)$. Several steps are necessary for defining the control algorithm development as follow.

\subsubsection{Step 1: generate the Input/Output data}

Three measurements with an electrical voltage from 0.8 to $5 \mathrm{kV}$ with $100 \mathrm{~V}$ step are performed to determine the frequency shift as a function of the applied electrical voltage $\Delta f=G(V)$ as shown in figure $13(\mathrm{a}, \mathrm{b})$. It is clearly observed from these figures that no membrane activation is made for voltage lower than $1.5 \mathrm{kV}$, which corresponds to a non linearities of the system. The effect appears for higher voltages. $50 \%$ of the obtained

(a)

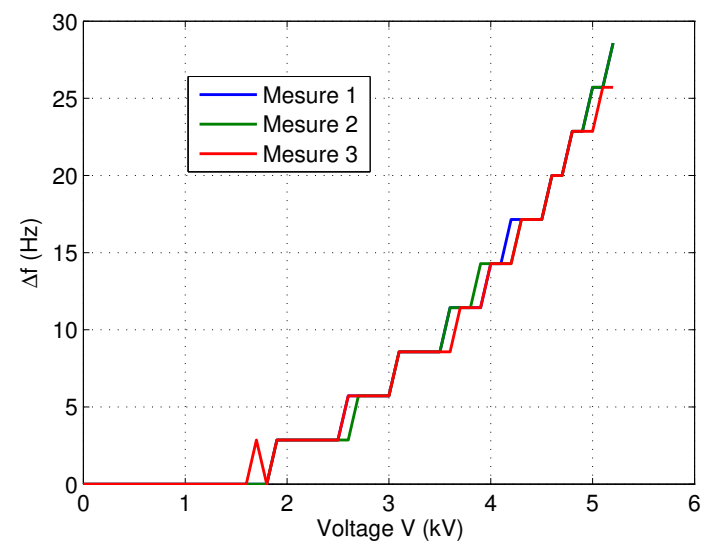

(b)

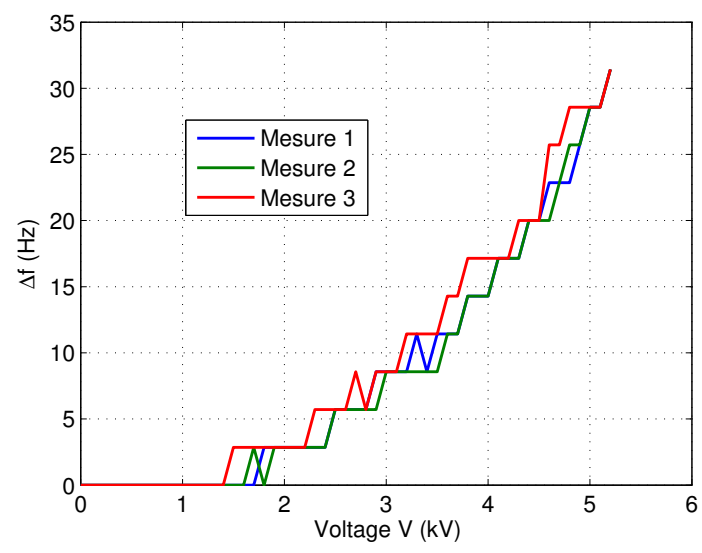

Figure 13. Evolution of the frequency shift $\Delta f(\mathrm{~Hz})$ as a function of the applied voltage $V(\mathrm{kV})$ for (a) $\lambda=3$ and (b) $\lambda=4$.

data (from measurements 1, 2 and 3) are used for the learning step, $25 \%$ for the test and $25 \%$ for validation.

\subsubsection{Step 2: linear function approach}

Assuming a quasi-static regime, the inverse non-linear function $G^{-1}$ that links the control voltage $V$ (to be applied) and the target frequency shift $\Delta f$ is established using the NN algorithm such as

$$
V=G^{-1}(\Delta f)
$$

\subsubsection{Step 3: Reference resonance frequency determination}

Once the function $G^{-1}$ assessed, a first measurement of absorption coefficient (black curve in figure 15) with white noise excitation for a zero voltage $(V=0 \mathrm{kV})$ is carried 
out. In order to determine the value of the first resonance $f_{0}$ (having a fixed value). Afterwards $f_{0}$ is used as the reference frequency in the following algorithm.

\subsubsection{Step 4: test of the control algorithm}

It consists, as depicted in figure 14 of exciting the device with a white noise combined with a tonal noise $f_{t}$. The latter being variable is chosen to be lower than that of the zero voltage resonator $\left(f_{0}\right)$. The difference $\Delta f$ between $f_{t}$ and $f_{0}$ leads to the calculated

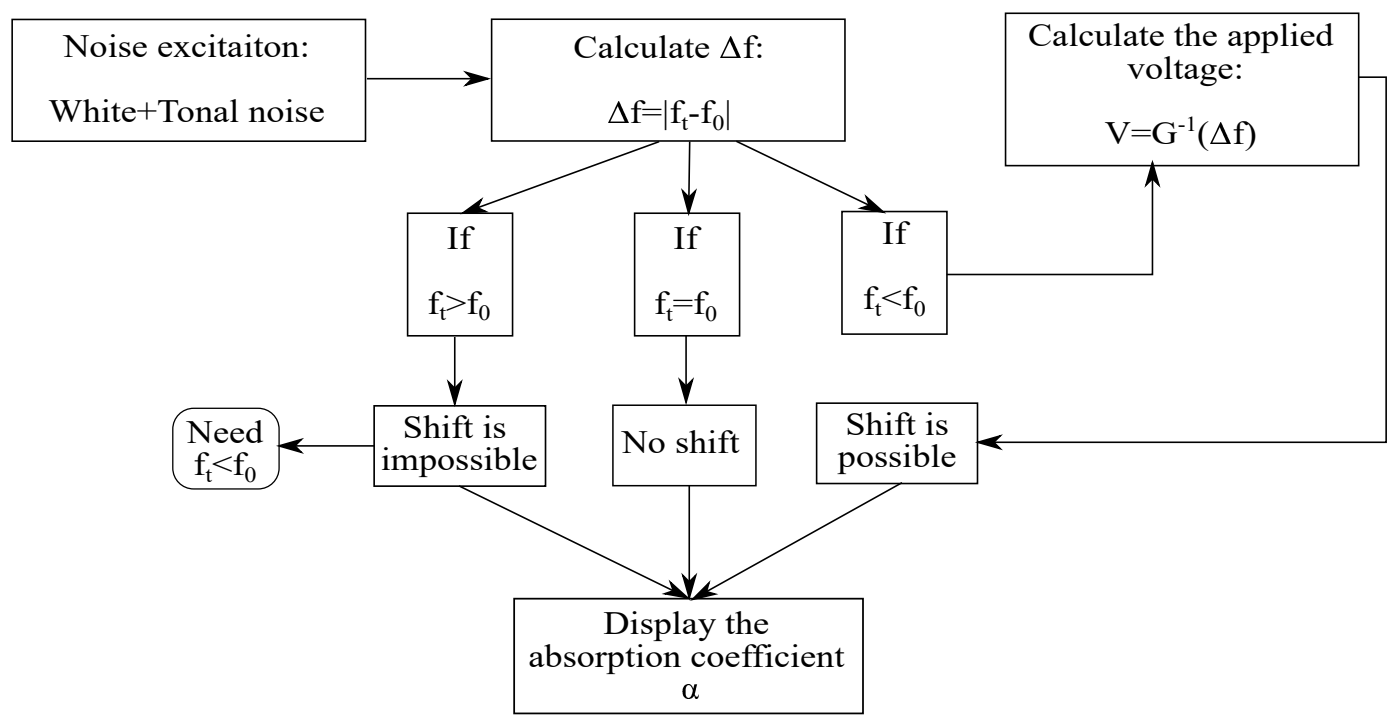

Figure 14. Diagram of the feedforward control applied to the adaptive HR based on EAP membrane (Resonance frequency shift process according to the desired command using the NN algorithm).

voltage value to be applied using the function $G^{-1}$ from step 2. An absorption curve is plotted and compared to the reference one in order to check the command accuracy.

\subsection{Results}

Acoustic absorption results obtained from the control are presented for $\lambda=3$ and 4 . 
(a)

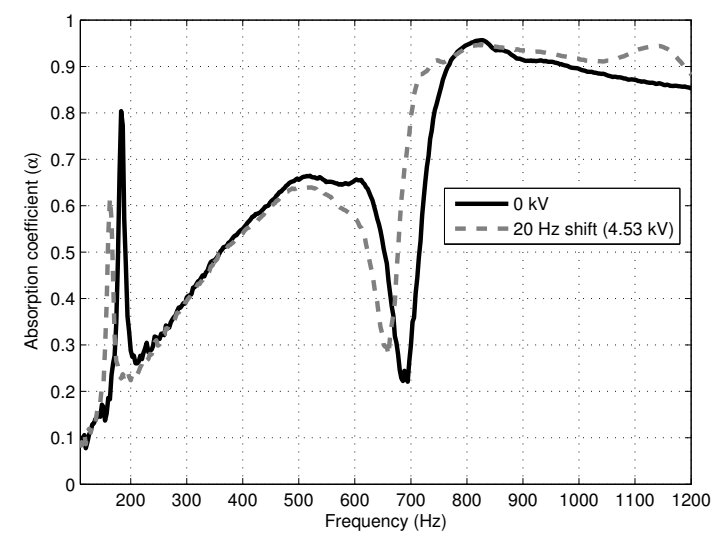

(b)

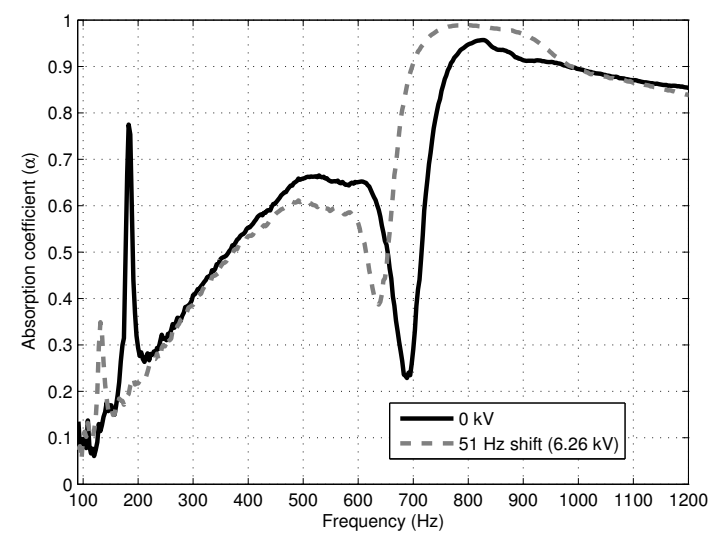

Figure 15. Feedforward algorithm response to the electroactive HR: frequency shift control (Case $\lambda=3$ ) for (a) $20 \mathrm{~Hz}(4.53 \mathrm{kV})$ and (b) $51 \mathrm{~Hz}(6.26 \mathrm{kV})$.

For $\lambda=3,20 \mathrm{~Hz}$ (corresponding to a tonal noise of $\left.f_{t}=164.5 \mathrm{~Hz}\right)$ then $51 \mathrm{~Hz}\left(f_{t}=135\right.$ $\mathrm{Hz}$ ) offsets are targeted. As shown in figure 15 (a, b), the command correctly estimates the adequate voltage to be applied $(4.53 \mathrm{kV}$ and $6.26 \mathrm{kV}$, respectively for the first and the second offset). Considering now $\lambda=4$, for $30 \mathrm{~Hz}$ corresponding to $f_{t}=156 \mathrm{~Hz}$, the $0 \mathrm{kV}\left(f_{0}=186 \mathrm{~Hz}\right)$ peak (black curve) is well shifted to $f=156 \mathrm{~Hz}$ as shown in figure 16 (a). On the other hand, the frequency is shifted to $121 \mathrm{~Hz}$ corresponding to a 65 $\mathrm{Hz}$ (with $5.93 \mathrm{kV}$ voltage). Thus, the developed command accurately adapts to the tonal excitation variation even for voltages not belonging to the set interval of step 1 $([0.8-5] \mathrm{kV})$, demonstrating the effectiveness of the proposed algorithm.

(a)

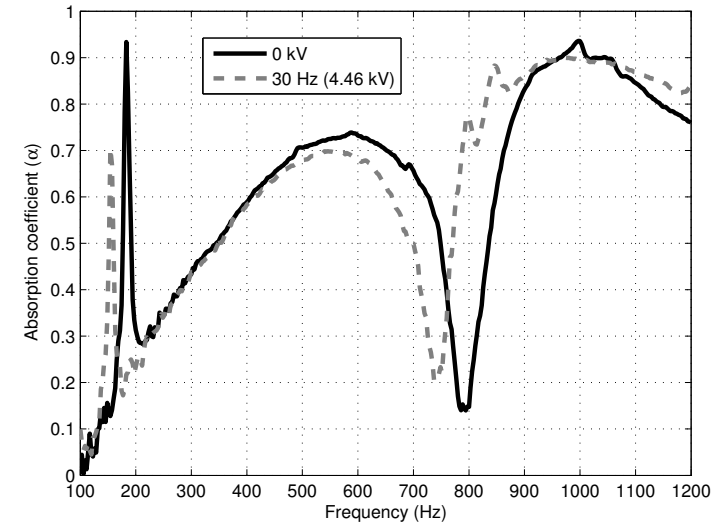

(b)

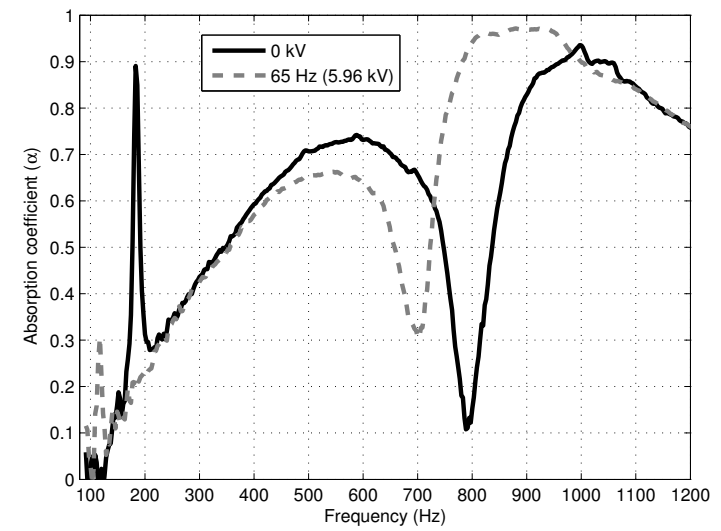

Figure 16. Feedforward algorithm response of the electroactive Helmholtz resonator: frequency shift control of $\lambda=4$ for (a) $30 \mathrm{~Hz}(4.46 \mathrm{kV})$ and (b) $65 \mathrm{~Hz}(5.96 \mathrm{kV})$.

For the two ratios $\lambda=3$ and $\lambda=4$, the frequency corresponding to the fall of the absorption coefficient (in the neighborhood of $800 \mathrm{~Hz}$ ) is also shifted, since it corresponds to the membrane resonance. A $6.26 \mathrm{kV}$ is needed to accomplish the $51 \mathrm{~Hz}$ shift for $\lambda=3$, or, $65 \mathrm{~Hz}$ shift $(\lambda=4)$, a lower voltage is applied. This is explained by the in-plane 
forces $T_{0}$ and membrane thickness differences that modify the electrical permittivity of the material. It can also be observed that resonance amplitudes decrease drastically when the shift control is important. Thus, for $65 \mathrm{~Hz}$, the first absorption resonance amplitude is decreased by $59 \%$ ( $89 \%$ amplitude at $0 \mathrm{kV}$ and $30 \%$ at $6.26 \mathrm{kV}$ ) which is in accordance with the membrane damping analysis presented in Section 2.3.

Giving the obtained results, the NN algorithm based control seems to be very effective for adaptive HR response to the acoustic tonal excitation variation.

\section{Conclusion}

In this paper, the acoustic response of an electroactive membraned Helmholtz resonator embedded in melamine foam has been investigated numerically. Experimental validation have shows a good agreement between measurements and simulations, which definitely demonstrates the effectiveness of the proposed model.

Results for absorption and transmission loss have shown the potential of the electroactive polymer membrane integration to control the Helmholtz resonator frequency by varying the applied voltage. In addition to the Helmholtz type resonance, other peaks are observed at higher frequencies resulting from the membrane vibration. A drop is observed in acoustic absorption, while a high amplitude peak effect is obtained in trans-

mission loss. The neural network algorithm allows the control of the resonance shift as function of the tonal acoustic excitation variation with a high accuracy. In the absence of disturbance, control by neural networks may be sufficient to obtain adaptive Helmholtz resonator and could open the control of the proposed system by taking into account the variation of conditions (temperature, humidity, etc.).

For future applications, and although resonators provide control over a narrow band, the combination of multiple tuned resonator at multiple frequencies could provide a potential solution for broadband control.

\section{Acknowledgments}

The authors would like to thank the National Sciences and Engineering Research Council of Canada (NSERC) for providing financial support. It has been performed in cooperation with the Labex ACTION program (ANR-11-LABX-0001-01).

\section{References}

[1] J. Allard and N. Atalla, Propagation of Sound in Porous Media: Modelling Sound Absorbing Materials 2e. John Wiley \& Sons, 2009.

[2] H. Wen-chao and N. Chung-fai, "Sound insulation improvement using honeycomb sandwich panels," Applied Acoustics, vol. 53, no. 1-3, pp. 163-177, 1998 . doi: https://doi.org/10.1016\%2Fs0003-682x\%2897\%2900033-9 
[3] M. Han and H. Myonghyon, "Sound reduction by a Helmholtz resonator," 2008.

[4] U. Ingard, "On the theory and design of acoustic resonators," The Journal of the acoustical society of America, vol. 25, no. 6, pp. 1037-1061, 1953. doi: https://doi.org/10.1121\%2F1.1907235

[5] I. Hughes and A. Dowling, "The absorption of sound by perforated linings," Journal of Fluid Mechanics, vol. 218, pp. 299-335, 1990.

[6] R. Chanaud, "Effects of geometry on the resonance frequency of Helmholtz resonators," Journal of Sound and Vibration, vol. 178, no. 3, pp. 337-348, 1994. doi: https://doi.org/10.1006\%2Fjsvi.1994.1490

[7] K. Nagaya, Y. Hano, and A. Suda, "Silencer consisting of two-stage helmholtz resonator with autotuning control," the Journal of the Acoustical Society of America, vol. 110, no. 1, pp. 289-295, 2001. doi: https://doi.org/10.1121\%2F1.1370524

[8] J. S. Lamancusa, "An actively tuned, passive muffler system for engine silencing.," vol. 126, no. 1, pp. pp. 313-318, 1987.

[9] J. M. De Bedout, Adaptive-passive noise control with self-tuning Helmholtz resonators. PhD thesis, Purdue University, 1996. doi: https://doi.org/10.1006\%2Fjsvi.1996.0796

[10] S. B. Horowitz, Design and characterization of compliant backplate Helmholtz resonators. PhD thesis, University of Florida, 2001. doi: https://doi.org/10.2514\%2F6.2002-666,

[11] F. Liu, S. Horowitz, T. Nishida, L. Cattafesta, and M. Sheplak, "A tunable electromechanical Helmholtz resonator," aiaa paper, vol. 3145, p. 2003, 2003.

[12] D.-F. Ruan and E.-Y. Zhang, "Effect of compliant-diaphragm geometry on the transmission loss characteristics of Helmholtz resonators," Noise 83 Vibration Worldwide, vol. 45, no. 11, pp. 9-15, 2014. doi: https://doi.org/10.1260\%2F0957-4565.45.11.9

[13] J. Yuan, "Active Helmholtz resonator with positive real impedance," Journal of Vibration and Acoustics, vol. 129, no. 1, pp. 94-100, 2007. doi: https://doi.org/10.1115\%2F1.2345678

[14] Y. Yun and C. Mak, "The theoretical fundamentals of an adaptive active control using periodic Helmholtz resonators for duct-borne transmission noise in ventilation systems," Building Services Engineering Research and Technology, vol. 34, no. 2, pp. 195-201, 2013. doi: https://doi.org/10.1177\%2F0143624410389578

[15] X. Zhao and Z. Suo, "Theory of dielectric elastomers capable of giant deformation of actuation," Physical review letters, vol. 104, no. 17, p. 178302, 2010. doi: https://doi.org/10.1103\%2Fphysrevlett.104.178302

[16] 3M Industrial Adhesives and Tapes Division, 3M VHB Tape Specialty Tapes, 2015.

[17] Y. Liu, L. Liu, Z. Zhang, and J. Leng, "Dielectric elastomer film actuators: characterization, experiment and analysis," Smart Materials and Structures, vol. 18, p. 095024, jul 2009. doi: https://doi.org/10.1088\%2F0964-1726\%2F18\%2F9\%2F095024

[18] G. Zhang, J. Gaspar, V. Chu, and J. Conde, "Electrostatically actuated polymer microresonators," Applied Physics Letters, vol. 87, no. 10, p. 104104, 2005. doi: https://doi.org/10.1063\%2F1.2040009

[19] P. Dubois, S. Rosset, M. Niklaus, M. Dadras, and H. Shea, "Voltage control of the resonance frequency of dielectric electroactive polymer (deap) membranes," Journal of Microelectromechanical Systems, vol. 17, no. 5, pp. 1072-1081, 2008. doi: https://doi.org/10.1109\%2Fjmems.2008.927741

[20] L. Liu, Y. Liu, X. Luo, B. Li, and J. Leng, "Electromechanical instability and snap-through instability of dielectric elastomers undergoing polarization saturation," Mechanics of Materials, vol. 55, pp. 60-72, dec 2012. doi: https://doi.org/10.1016\%2Fj.mechmat.2012.07.009,

[21] J. Zhu, S. Cai, and Z. Suo, "Resonant behavior of a membrane of a dielectric elastomer," International Journal of Solids and Structures, vol. 47, no. 24, pp. 3254-3262, 2010. doi: https://doi.org/10.1016\%2Fj.ijsolstr.2010.08.008

[22] J. Den Hartog and J. D. H. fourth edition Mechanical, Vibrations. McGraw-Hill Book Company, Inc., New York, 1956.

[23] L. Liu, Y. Liu, K. Yu, and J. Leng, "Thermoelectromechanical stability of dielectric elastomers 
undergoing temperature variation," Mechanics of Materials, vol. 72, pp. 33-45, may 2014. doi: https://doi.org/10.1016\%2Fj.mechmat.2013.05.013

[24] Y. Bar-Cohen, Electroactive polymer (EAP) actuators as artificial muscles: reality, potential, and challenges, vol. 136. SPIE press, 2004. doi: https://doi.org/10.1117\%2F3.547465.ch21

[25] H. Monner, "Smart materials for active noise and vibration reduction," NOVEM: Noise and Vibration: Emerging Methods, Saint-Raphal, France, 2005.

[26] M. Shahinpoor and K. J. Kim, "Ionic polymer-metal composites: Iv. industrial and medical applications," Smart materials and structures, vol. 14, no. 1, p. 197, 2005. doi: https://doi.org/10.1088\%2F0964-1726\%2F14\%2F1\%2F020

[27] P. Brochu and Q. Pei, "Advances in dielectric elastomers for actuators and artificial muscles," Macromolecular rapid communications, vol. 31, no. 1, pp. 10-36, 2010. doi: https://doi.org/10.1002\%2Fmarc.200900425

[28] Y. Liu, L. Liu, Z. Zhang, Y. Jiao, S. Sun, and J. Leng, "Analysis and manufacture of an energy harvester based on a mooney-rivlin-type dielectric elastomer," EPL (Europhysics Letters), vol. 90, p. 36004, may 2010. doi: https://doi.org/10.1209\%2F0295-5075\%2F90\%2F36004

[29] X. Lv, L. Liu, Y. Liu, and J. Leng, "Dielectric elastomer energy harvesting: maximal converted energy, viscoelastic dissipation and a wave power generator," Smart Materials and Structures, vol. 24, p. 115036, oct 2015. doi: https://doi.org/10.1088\%2F0964-1726\%2F24\%2F11\%2F115036

[30] K. Hochradel, S. Rupitsch, A. Sutor, R. Lerch, D. Vu, and P. Steinmann, "Dynamic performance of dielectric elastomers utilized as acoustic actuators," Applied Physics A, vol. 107, no. 3, pp. 531538, 2012. doi: https://doi.org/10.1007\%2Fs00339-012-6837-2

[31] Z. Lu, H. Godaba, Y. Cui, C. C. Foo, M. Debiasi, and J. Zhu, "An electronically tunable duct silencer using dielectric elastomer actuators," The Journal of the Acoustical Society of America, vol. 138, no. 3, pp. EL236-EL241, 2015. doi: https://doi.org/10.1117\%2F12.2044632

[32] X. Yu, Z. Lu, F. Cui, L. Cheng, and Y. Cui, "Tunable acoustic metamaterial with an array of resonators actuated by dielectric elastomer," Extreme Mechanics Letters, 2016.doi: https://doi.org/10.1016\%2Fj.eml.2016.07.003

[33] X. Yu, Z. Lu, L. Cheng, and F. Cui, "Vibroacoustic modeling of an acoustic resonator tuned by dielectric elastomer membrane with voltage control," Journal of Sound and Vibration, vol. 387, pp. 114-126, 2017. doi: https://doi.org/10.1016\%2Fj.jsv.2016.10.022

[34] M. Ouisse, L. Maxit, C. Cacciolati, and J.-L. Guyader, "Patch transfer functions as a tool to couple linear acoustic problems," Journal of vibration and acoustics, vol. 127, no. 5, pp. 458-466, 2005. doi: https://doi.org/10.1115\%2F1.2013302

[35] O. Doutres, N. Atalla, and H. Osman, "Transfer matrix modeling and experimental validation of cellular porous material with resonant inclusions," The Journal of the Acoustical Society of America, vol. 137, no. 6, pp. 3502-3513, 2015. doi: https://doi.org/10.1121\%2F1.4921027

[36] A. Abbad, K. Jaboviste, M. Ouisse, and N. Dauchez, "Acoustic performances of silicone foams for sound absorption," Journal of Cellular Plastics, p. 0021955X17732305, 2017. doi: https://doi.org/10.1177\%2F0021955x17732305

[37] S. MARBURG, "Six boundary elemnts per wavelength: is that enough?," Journal of Computational Acoustics, vol. 10, pp. 25-51, mar 2002. doi: https://doi.org/10.1142\%2Fs0218396x02001401

[38] V. Romero-García, G. Theocharis, O. Richoux, and V. Pagneux, "Use of complex frequency plane to design broadband and sub-wavelength absorbers," The Journal of the Acoustical Society of America, vol. 139, no. 6, pp. 3395-3403, 2016. doi: https://doi.org/10.1121\%2F1.4950708

[39] M. L. Munjal, M. Vorländer, P. Költzsch, M. Ochmann, A. Cummings, W. Maysenhölder, W. Arnold, and F. Mechel, Formulas of acoustics. Springer Science \& Business Media, 2008.

[40] M. R. Stinson, "The propagation of plane sound waves in narrow and wide circular tubes, and generalization to uniform tubes of arbitrary cross-sectional shape," The Journal of the Acoustical Society of America, vol. 89, no. 2, pp. 550-558, 1991. doi: https://doi.org/10.1121\%2F1.400379

[41] R. Ogden, "Large deformation isotropic elasticity-on the correlation of theory and experiment for incompressible rubberlike solids," in Proceedings of the Royal Society of London A: 
Mathematical, Physical and Engineering Sciences, vol. 326, pp. 565-584, The Royal Society, 1972. doi: https://doi.org/10.5254\%2F1.3542910

[42] G. Kofod, P. Sommer-Larsen, R. Kornbluh, and R. Pelrine, "Actuation response of polyacrylate dielectric elastomers," Journal of intelligent material systems and structures, vol. 14, no. 12, pp. 787-793, 2003. doi: https://doi.org/10.1117\%2F12.432638

[43] J. Chung and D. Blaser, "Transfer function method of measuring in-duct acoustic properties. ii. experiment," The Journal of the Acoustical Society of America, vol. 68, no. 3, pp. 914-921, 1980. doi: https://doi.org/10.1121\%2F1.384779

[44] Y. Salissou, R. Panneton, and O. Doutres, "Complement to standard method for measuring normal incidence sound transmission loss with three microphones," The Journal of the Acoustical Society of America, vol. 131, no. 3, pp. EL216-EL222, 2012. doi: https://doi.org/10.1121\%2F1.3681016

[45] D. M. Photiadis, "The effect of wall elasticity on the properties of a Helmholtz resonator," The Journal of the Acoustical Society of America, vol. 90, no. 2, pp. 1188-1190, 1991. doi: https://doi.org/10.1121\%2F1.2028905

[46] A. Norris and G. Wickham, "Elastic Helmholtz resonators," The Journal of the Acoustical Society of America, vol. 93, no. 2, pp. 617-630, 1993. doi: https://doi.org/10.1121\%2F1.404517

[47] S. S. Nudehi, G. S. Duncan, and U. Farooq, "Modeling and experimental investigation of a Helmholtz resonator with a flexible plate," Journal of Vibration and Acoustics, vol. 135, no. 4, p. 041102, 2013. doi: https://doi.org/10.1115\%2F1.4023810

[48] A. Abbad, "Numerical investigations on a tunable Helmholtz resonator: Integration of a passive polymer membrane in a Helmholtz resonator," tech. rep., SAE Technical Paper, 2016. doi: https://doi.org/10.4271\%2F2016-01-1842

[49] "Matlab neural network toolbox,", 2015. doi: http://doi.org/10.1002/0470848944.hsa251

[50] M. H. Beale, M. T. Hagan, and H. B. Demuth, "Neural network toolbox user's guide," The Mathworks Inc, 1992. 